\title{
Métropolisation et système de villes en Pologne depuis 1960
}

Metropolisation and system of cities in Poland since 1960

Metropolenbildung und das System der Städte in Polen seit 1960

\section{Natalia Zdanowska}

\section{OpenEdition}

\section{Journals}

Electronic version

URL: http://journals.openedition.org/rge/5891

ISSN: 2108-6478

\section{Publisher}

Association des géographes de l'Est

\section{Printed version}

Date of publication: 1 December 2016

ISSN: 0035-3213

\section{Electronic reference}

Natalia Zdanowska, « Métropolisation et système de villes en Pologne depuis 1960 », Revue

Géographique de l'Est [Online], vol. 56 / n³-4 | 2016, Online since 29 December 2016, connection on 08 September 2020. URL : http://journals.openedition.org/rge/5891

This text was automatically generated on 8 September 2020.

Tous droits réservés 


\title{
Métropolisation et système de villes en Pologne depuis 1960
}

\author{
Metropolisation and system of cities in Poland since 1960 \\ Metropolenbildung und das System der Städte in Polen seit 1960
}

Natalia Zdanowska

1 Le processus de métropolisation peut se définir comme la concentration de la population et des fonctions de décisions économiques majeures dans les métropoles, notamment à l'échelon du système-monde (Sassen, 1991 ; Taylor, 2004 ; Vandermotten et al., 2010). «Une métropole est une ville, voire même plus qu'une ville $~^{1}$ (BourdeauLepage, Huriot, 2005, p. 257). Elle désigne une ville exerçant une domination politique ou économique sur un territoire environnant (Bretagnolle, 2013). Véritables nœuds des réseaux de transport et d'informations, les métropoles contribuent au processus de sélection et de concentration géographiques des activités de contrôle, y compris au niveau international (Bretagnolle, Le Goix, Vacchiani-Marcuzzo, 2011). En Europe Centrale et Orientale ${ }^{2}$, la métropolisation s'est réellement mise en place après la chute du mur de Berlin (Pyka, 2011 ; Jałowiecki, 2005). Dès le début des années 1990, certaines villes centre-orientales se sont dotées de caractéristiques métropolitaines dans le sens, où elles sont devenues plus connectées avec des métropoles étrangères qu'avec les villes de proximité nationale (Jałowiecki, 2005 ; Korcelli-Olejniczak, 2004). Les travaux du groupe $\mathrm{GaWC}^{3}$, ont identifié Varsovie, Prague, Budapest et Bucarest parmi les vingt métropoles les plus connectées au sein des réseaux européens d'entreprises du secteur des services (Taylor, 2003). Cette période, qui correspond à une mondialisation intense de l'économie, s'est accompagnée d'une mise en réseau des villes au niveau mondial (Michalet, 2007). Les métropoles des pays anciennement industrialisés, devenues des "vitrines de la mondialisation» (Bretagnolle et al., 2011), se sont inscrites dans une logique de compétition pour attirer le plus grand nombre d'activités qualifiées et dynamiques (Pumain, Rozenblat, 2007). Ainsi, la métropolisation en Europe Centrale et Orientale a été exacerbée par son insertion directe à l'échelon du monde. Ce processus qui s'opère sur une période beaucoup plus courtes que dans les pays à système libéral (Pyka, 2011), a pu avoir un impact sur la manière sélective dont se sont opérées les 
transformations parmi les villes postsocialistes de l'Europe centre-orientale après 1989 (Bourdeau-Lepage, 2004a ; Zdanowska, 2015).

2 À l'échelle d'un système de villes national, entendu comme un «ensemble de villes rendues interdépendantes dans leurs évolutions » (Pumain, 2007), la métropolisation concerne généralement les capitales, mais parfois également d'autres villes de taille importante (Cattan, Saint-Julien, 1998). L'influence de ce processus sur la forme du système de villes en Pologne présente un intérêt tout particulier. En effet son système urbain se caractérise par une capitale peu prédominante sur les autres villes en termes démographiques. Il s'agit d'un héritage qui remonte aux partages du pays entre Empires autrichien, prussien et russe de 1772 à 1918 (Trzeciakowski, 1981). L'évolution de systèmes urbains distincts selon les empires a contribué au développement de nombreuses villes moyennes surtout à l'Ouest de la Pologne actuelle (WĘcławowicz, 2010). De nos jours, même si les plus grandes villes polonaises sont loin d'avoir un rôle tel que celui de villes mondiales comme New York, Tokyo et Londres (Sassen, 1991), au moins six à part la capitale ${ }^{4}$ détiennent des fonctions métropolitaines ${ }^{5}$ dans le domaine des services financiers (Bourdeau-Lepage, 2004), de l'informatique ou de l'industrie (Pyka, 2011).

3 Le concept de «métropole » apparaît dans les documents officiels polonais qu'à partir des années $2000^{6}$ (Gorzelak et al., 2009). A part Varsovie, d'autres villes sont présentées comme des métropoles potentielles dans un futur proche (Gdańsk, Poznań, Cracovie) ou plus lointain (Szczecin, Wrocław, Łódź, Katowice, Lublin, Białystok, Rzeszów). Le plan national de développement 2007-2013, met en évidence une dynamique métropolitaine à Varsovie, Cracovie, Poznań, Wrocław, la conurbation silésienne ${ }^{8}$, Szczecin et Łódź (Gorzelak et al., 2009).

4 Une liste de métropoles mondiales est difficile à déterminer, car des données comparables ne sont pas disponibles à l'échelon du monde (Bretagnolle, 2015). En Europe, de nombreux classements existent et combinent des facteurs relatifs à l'attractivité économique et à l'accessibilité. Selon le classement des MEGA ${ }^{9}$, provenant notamment des travaux de P. J. Taylor et de F. Dobruszkes, cinq villes sont des métropoles en Pologne (Varsovie, conurbation silésienne, Cracovie, Lódź et Gdańsk) (ESPON 1.4.3, 2007). Cependant, d'autres travaux en identifient sept (Varsovie, conurbation Silésienne, Cracovie, Poznań, Gdańsk-Gdynia-Sopot, Wrocław, Łódź) (Pyka, 2011 ; Gorzelak et al., 2009).

5 L'objectif de cet article n'est pas d'identifier les métropoles polonaises. Nous cherchons à savoir comment la métropolisation se traduit depuis 1989 en termes d'évolution de la hiérarchie urbaine des villes polonaises et de leur répartition au sein de l'espace national. A-t-elle concentré les flux économiques mondiaux orientés principalement vers les grandes villes ou également vers des villes de tailles moins importantes?

6 Plusieurs critères ont été identifiés pour qualifier les systèmes de villes nationaux de l'Union européenne comme polycentriques ou monocentriques (ESPON 1.1.1, 200510). La polycentricité est proposée dès le milieu des années 1990, en tant que modèle pour assurer le développement régional de manière uniforme sur l'ensemble du territoire (Geppert, 2009). Au niveau national elle se caractérise par la présence de plusieurs villes d'importance équivalente plutôt que par une simple domination d'une seule ville. Elle est opposée à la monocentricité qui elle reflète l'idée d'une concentration en un seul centre « de la gestion territoriale et de l'offre de services» (ORATE 1.1.1, 2005, p. $\left.1^{11}\right)$. 
7 Plus précisément le projet ESPON 1.1.1 définit la polycentricité par deux aspects : la morphologie et les relations entre villes (ESPON 1.1.1, 2005). Le critère morphologique repose sur la forme de la distribution statistique des tailles de villes et sur la répartition des villes selon leur taille dans le territoire. Une distribution rang-taille « horizontale est plus polycentrique qu'une distribution pentue, et un système urbain polycentrique ne peut pas être dominé par une seule grande ville» (ORATE 1.1.1, p. 3). En ce qui concerne la répartition des villes, « une distribution uniforme des villes sur le territoire convient mieux à un système urbain polycentrique qu'une distribution très polarisée où les principales villes sont toutes regroupées sur une partie du territoire » (ORATE 1.1.1, 2005, p. 3). A propos du critère reposant sur les relations entre villes, il est spécifié que "dans un système polycentrique, les petites villes bénéficient autant que les grandes d'une bonne accessibilité » ce qui peut être envisagé dans le cadre de réseaux de flux et de coopération (ORATE 1.1.1, 2005, p. 3).

8 A partir de chacune de ces trois dimensions, un index de polycentricité a été élaboré pour les pays de l'Union européenne. Ainsi, selon le projet ESPON 1.1.1, certains pays (Grèce, Irlande, Lettonie) sont caractérisés par une monocentricité élevée ou une polycentricité moyenne (France, Royaume Uni), voire forte (Allemagne, Pologne) (ESPON 1.1.1,2005). La Pologne serait le deuxième pays après la Slovénie en Europe Centrale et Orientale à présenter une forme polycentrique. Cependant, selon certains travaux (Komornicki et al. 2013), portant sur les MEGA en Pologne, l'hypothèse d'une polycentricité élevée en Pologne serait à rediscuter au vu de l'hypertrophie économique de la capitale (Śleszyński, 2007 ; 2013).

9 Nous proposons d'étudier ici le lien entre la métropolisation et la dynamique démographique et économique des villes polonaises ${ }^{12}$. Nous procèderons d'abord à l'analyse de la hiérarchie urbaine et de la distribution spatiale des villes dans l'espace national ${ }^{13}$ à partir de la base de données TRADEVE (Bretagnolle et al., 2016) sur les délimitations et populations des agglomérations urbaines en Europe de 1961-2011 ${ }^{14}$. Son intérêt est de pouvoir saisir l'évolution du système de villes avant et après le début de la métropolisation en Pologne avec une même définition de l'agglomération. En outre, l'impact du phénomène métropolitain sur les relations fonctionnelles des villes polonaises dans les réseaux économiques a été étudié grâce aux bases de données PołĄczenia Świat (Connections Monde) (Śleszyński, 2007) et Orbis du Bureau Van Dijk (Rozenblat, Di Lello, 2014) ${ }^{15}$. Ces dernières permettent de localiser dans les villes toutes les entreprises polonaises dont le capital est contrôlé par les institutions étrangères en 2004 et 2013. Une autre source a été la base de données Handel Zagraniczny (Commerce International) sur les échanges commerciaux permettant d'une manière assez rare en Europe Centrale et Orientale de cartographier les exportations depuis les villes polonaises vers les pays de monde en 2005 et 2011 (Komornicki, Szejgiec, 2015) ${ }^{16}$.

\section{Distribution des tailles des villes en Pologne et son évolution depuis 1961}

\section{A. Base de données TRADEVE}

10 La définition de la ville diffère selon les pays en Europe. Certains ont pu adopter une approche tantôt juridique (octroi d'un statut), quantitative (seuil statistique de population), morphologique (caractère plus ou moins groupé de l'habitat), ou plus tard 
fonctionnelle (liée aux déplacements domicile-travail) (Bretagnolle, 2009). La définition de la ville en Pologne est juridique. Le statut de ville est octroyé par décision du Conseil des ministres (Ustawa o SamorzĄdzie Gminnym z dnia 8 marca 1990 r. ${ }^{17}$ ). Différents critères de taille de la localité et d'état de l'infrastructure rentrent en jeu, mais sont très flexibles et subjectifs (Zborowski, 2009). Pour étudier le phénomène métropolitain qui dépasse souvent les limites administratives des communes-centres (Bretagnolle, 2013), il semble pertinent de se tourner vers d'autres définitions comme celle de l'agglomération ou de l'aire fonctionnelle (Bretagnolle, 2009).

11 Plusieurs bases de données sur les aires fonctionnelles ont été générées en Europe, entre autres les Functional Urban Areas ${ }^{18}$ (Peeters, 2011). Des experts nationaux ont identifié 151 FUA en Pologne (Śleszyński, 2013).Le problème est que les bases de données sont construites pour les plus grandes villes (plus de 50000 habitants dans le cas des Functional Urban Areas), limitant le nombre d'entités pour l'examen de l'évolution de la hiérarchie urbaine. De plus, ces types de bases ne sont généralement disponibles que depuis les années 2000, ce qui ne permet pas de remonter assez loin dans le temps pour l'étude de la métropolisation en Pologne. Pour cette raison, le concept d'agglomération paraît plus intéressant.

De nombreux critères morphologiques permettent de définir l'agglomération, notamment la continuité du bâti ou la densité minimale de population (Bretagnolle, 2009). Certaines bases de données utilisent le critère de densité minimale comme les Cities (Dijkstra, Poelman, 2012), ou encore les Morphological Urban Areas (MUA) (Peteers, 2011). Les Urban Morphological Zones de 2000 de l'Agence Européenne de l'Environnement (European Environment Agency) (EEA, 2002) sont quant à elles définies selon la continuité du bâti. En effet, La base des UMZ de 2000 a été construite selon une distance maximale de 200 mètres entre les tâches bâties (EEA, 2002). Cette définition est donc proche de celles utilisées par d'autres pays européens (Le Gléau et al., 1996) comme la France, dans le cas des unités urbaines, le Danemark ou la Suède (Bretagnolle et al., 2012).

13 Les UMZ de 2000, constituent un outil de travail robuste pouvant être appliqué en Europe Centrale et Orientale, où il n'existe pas de bases morphologiques nationales (Guérois et al., 2012).

14 Dans le cadre du projet TRADEVE, nous avons construit entre 1961 et 2011 une définition d'agglomération constituée de une ou plusieurs LAU2 ${ }^{19}$ en Pologne, en partant de la délimitation des UMZ en 2000 et en combinant des critères de contiguïté du bâti et de population minimale pour rétropoler cette délimitation dans le temps (Bretagnolle et al., 2016). Certaines bases utilisent une population minimale de 10000 habitants (Moriconi-Ebrard, 1994), 20000 (Vandermotten et al, 1999) ou 50000 habitants (Parr, 2007), d'autres un critère de densité minimale de 650 habitants par km² comme dans le cas des Morphological Urban Areas (MUA) (Peeters, 2011). Après plusieurs tests réalisés au niveau de l'Europe, nous avons retenu un seuil de 2000 habitants pour la sélection des LAU2 contigus à l'intérieur du périmètre rétropolé (figure 1, carte 2), et de 10000 habitants pour la population totale des agglomérations formées à chaque date (figure 1 , carte 3$)^{20}$. 
Figure 1: Construction des agglomérations urbaines à partir des Urban Morphological Zones de 2000 : exemple théorique

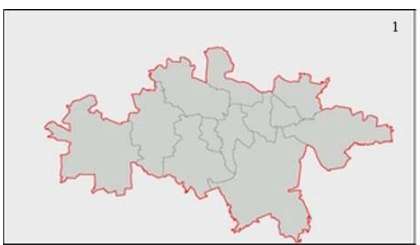

Temps $\mathrm{t}$

Une UMZ en 2000

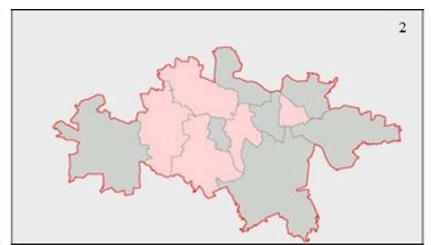

Temps t-1

Sélection des LAU2s urbaines en 1950

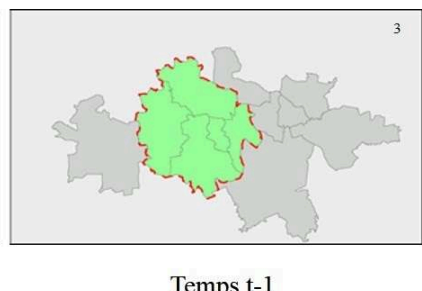

Temps t-1

Agrégation des LAU2s urbaines avec un traitement des espaces non contigus et des vides

Figure 2 : Délimitation des agglomérations urbaines en Pologne (1961-2011) ${ }^{21}$

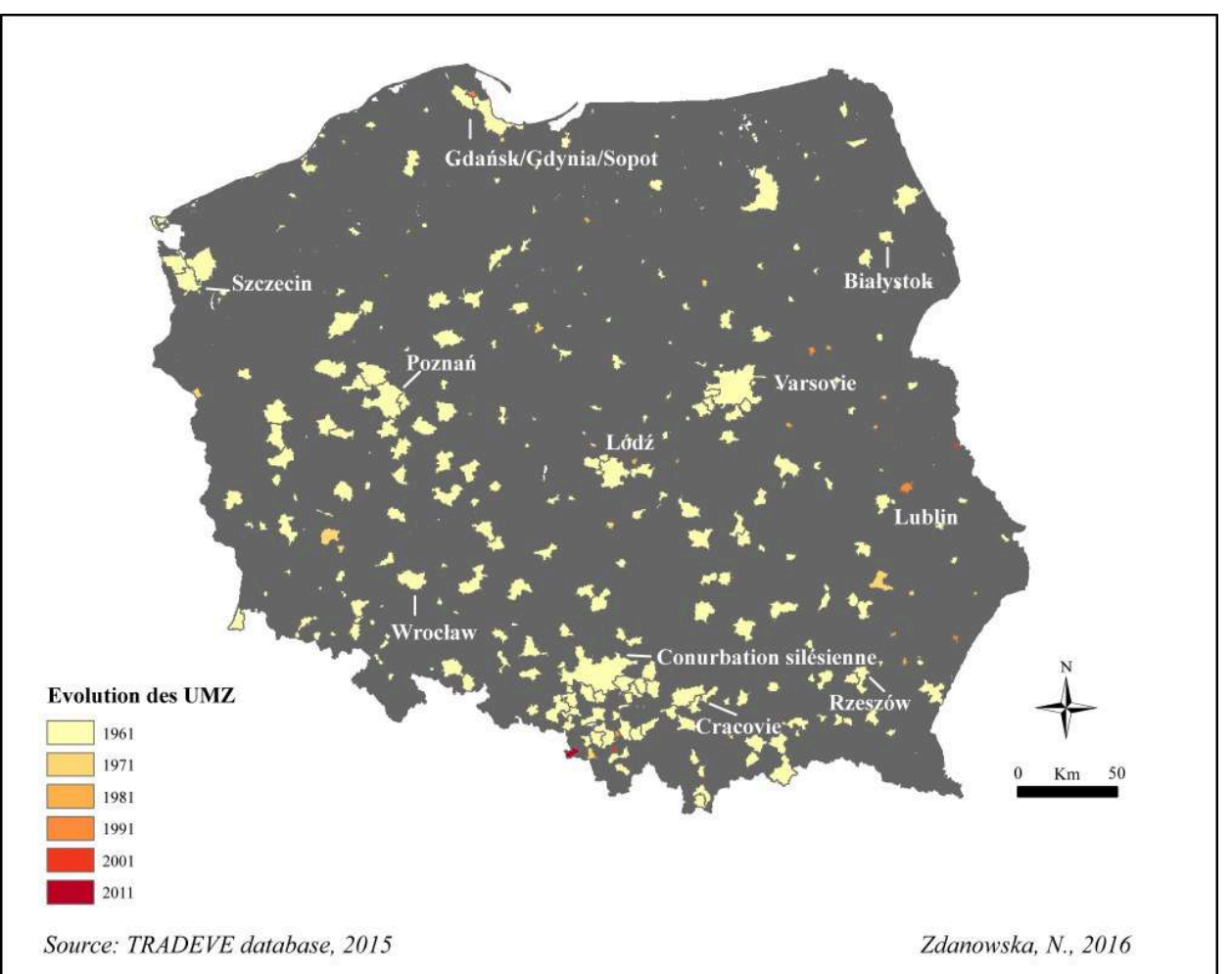

15 Comme le montre la figure 2, les délimitations des agglomérations urbaines morphologiques n'ont pas évolué en Pologne depuis 1961. Cela s'explique par le fait que l'étalement urbain a commencé dès la fin du 19ème siècle lors la construction des trains de banlieues (Lijewski, 1958) et a entrainé l'annexion progressive de LAU2 entières par les municipalités centrales. De cette manière les banlieues ont été absorbées progressivement par les centres (Bretagnolle et al., 2016). Depuis 1945, la période du socialisme s'est caractérisée par la restriction de l'étalement urbain dans les grandes villes et un contrôle de migrations entre elles (Zborowski, 2009). La base de données TRADEVE permet néanmoins d'observer l'apparition de nouvelles agglomérations dans le Sud-Est de Pologne à partir de 1991. 


\section{B. Analyse de l'évolution de la hiérarchie urbaine}

Afin d'entreprendre l'analyse de la hiérarchie urbaine, la distribution de la population des agglomérations polonaises a été représentée sous forme de graphiques rang-taille. Elle a été ensuite ajustée par une droite de régression, afin d'analyser l'évolution dans le temps de la valeur absolue de la pente de celle-ci. En effet, comparée dans le temps, la pente est un indicateur de variation de l'intensité de la hiérarchie urbaine (MoriconiEbrard, 1994).

Les résultats sur l'ensemble de la période 1961-2001 montrent l'absence de macrocéphalie (Bretagnolle et al., 2007) dans le système urbain polonais. En 2011, la première ville, Varsovie, comptait 2138493 habitants. La conurbation silésienne, deuxième ville, était peuplée de 1903454 habitants. De plus une persistance entre 1961 et 2011 des villes de taille importante peut être relevée, comme c'est le cas de Łódź ou Gdańsk (Pyka, 2011).

Figure 3 : Graphique rang-taille en 2011, évolution de la pente de la droite de régression et population des dix plus grandes agglomérations en Pologne (1961-2011)
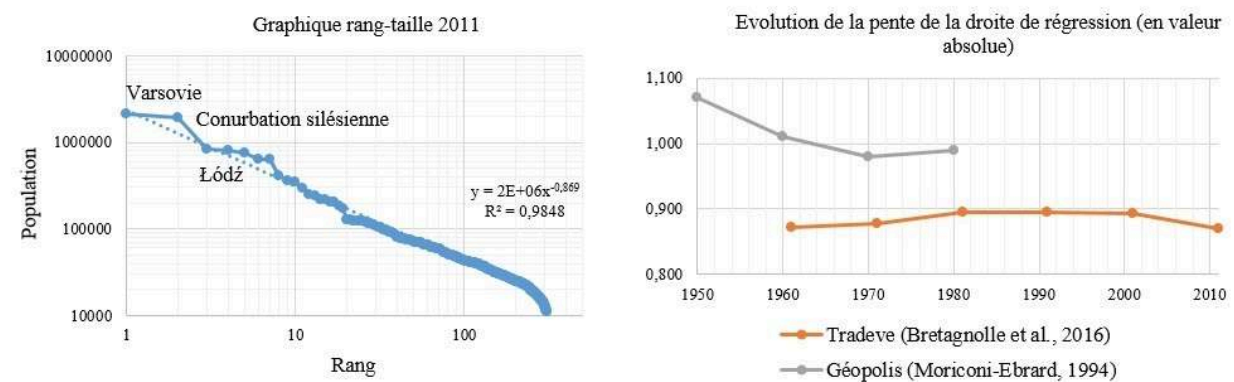

Population des 10 plus grandes agglomérations urbaines morphologiques

\begin{tabular}{|l|r|r|r|r|r|r|}
\hline & 1961 & 1971 & 1981 & 1991 & 2001 & 2011 \\
\hline Varsovie & 1402099 & 1629084 & 1888385 & 1995362 & 2043518 & 2138493 \\
\hline Conurbation silésienne & 1744590 & 1878289 & 2032253 & 2110314 & 1999472 & 1903454 \\
\hline Lódź & 798358 & 862784 & 932593 & 935078 & 878093 & 828971 \\
\hline Gdańsk/ Gdynia & 508172 & 642131 & 755939 & 796477 & 786383 & 794554 \\
\hline Cracovie & 502890 & 612227 & 704388 & 746429 & 745474 & 757304 \\
\hline Poznań & 445071 & 517372 & 597266 & 641928 & 646153 & 647305 \\
\hline Wrocław & 438975 & 533974 & 606043 & 636316 & 623552 & 629954 \\
\hline Szczecin & 271764 & 340705 & 390451 & 409308 & 402532 & 409933 \\
\hline Bydgoszcz & 225310 & 274935 & 342196 & 372578 & 368551 & 364037 \\
\hline Lublin & 184928 & 242148 & 302332 & 342588 & 346035 & 349029 \\
\hline
\end{tabular}

Source: base de données TRADEVE, 2015

Un résultat remarquable concerne l'augmentation de la valeur absolue du coefficient de la pente entre 1961 et 1991 de 0,871 à 0,895, puis sa diminution jusqu'à 0,869 en 2011 avec une légère accentuation de cette chute entre 2001 et 2011. Ces observations reflètent plusieurs phases de l'histoire polonaise. Les années 1960 correspondent à l'industrialisation forcée de l'ancien bassin de la Silésie menée par la République populaire de Pologne ( $\mathrm{PRL}^{22}$ ). A partir de 1953 une décentralisation de la conurbation et la construction d'habitations dans ses périphéries est entamée (Kotela, 1990) entrainant l'augmentation des emplois et de la population (figure 1). Cependant la transition après 1989 s'est accompagnée d'une crise économique, écologique et démographique de cette région mono-industrielle (Gwosdz, 2004). A partir de 1991, Varsovie devient pour la première fois depuis 1961 la ville la plus peuplée (figure 1). Ceci traduit un processus de rééquilibrage des populations, malgré le fait que sur l'ensemble de la période le 
système de ville polonais semble peu hiérarchisé. Les coefficients de la pente de la droite de régression de la base Géopolis (Moriconi-Ebrard, 1994) montrent des résultats similaires. Ces éléments permettent de confirmer le fait que le système de villes en Pologne est de plus en plus polycentrique du point de vue démographique, résultat similaire à celui d'autres études récentes (Gorzelak et al., 2009).

Ainsi, cette première analyse de la hiérarchie urbaine des villes en Pologne révèle une tendance vers moins d'inégalités entre les villes soit un système urbain devenu plus polycentrique depuis les années 1990.

\section{Analyse de la trame urbaine et son évolution depuis 1961}

Nous avons cartographié tous les dix ans le taux de croissance annuel moyen des agglomérations morphologiques polonaises, afin d'analyser l'évolution de la trame urbaine. Cette notion de trame fait référence à la localisation et la taille des villes, et désigne au sens large la manière dont les villes structurent le territoire, le maillent (Brunet et al., 1993 ; Saint-Julien, 1981). Nous cherchons à voir si la comparaison de la trame au cours du temps traduit spatialement la réduction des inégalités entre les villes observée dans la section précédente.

Figure 4 : Taux de croissance annuel moyen des agglomérations en Pologne pour les périodes $1961-1971,1971-1981,1981-1991,1991-2001$ et $2001-2011^{23}$
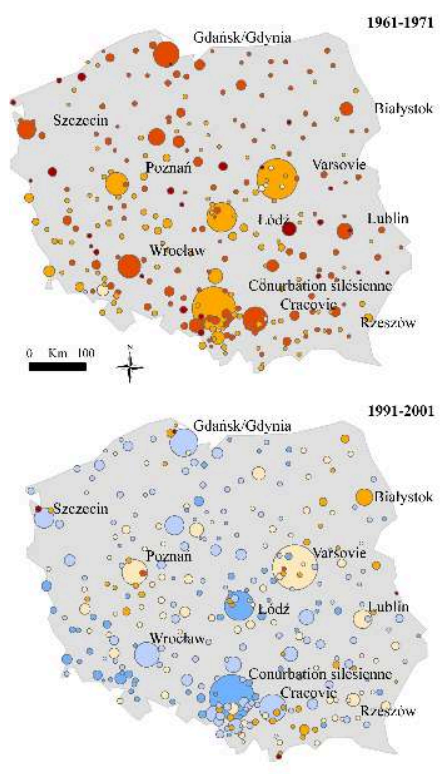

Source: base de données TR.ADEVE, 2015
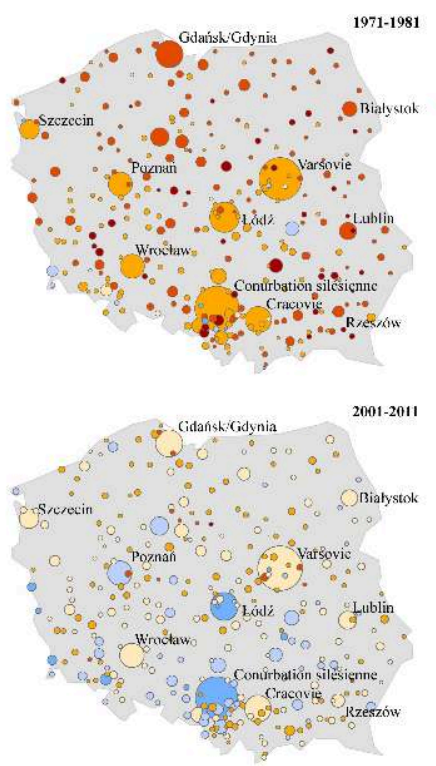

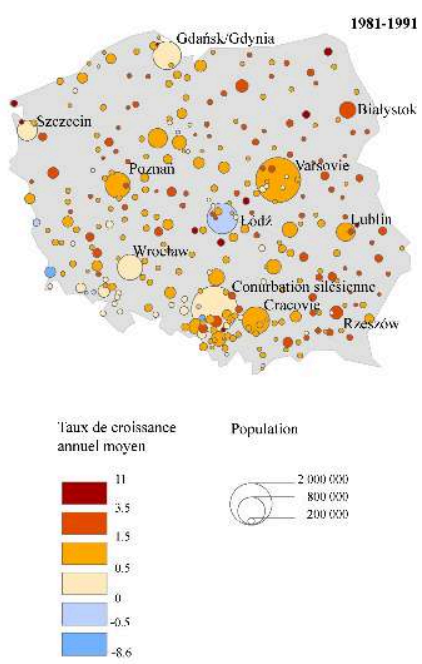

Zdanowska. N. 2016

D'après la figure 5, le système de villes apparaît comme déséquilibré entre l'Est et l'Ouest du pays en termes de répartition de grandes villes. Les zones à l'Ouest sont beaucoup plus denses qu'à l'Est (Gorzelak et al., 2009). En dehors de Varsovie, il n'y a pas de grandes villes et très peu de villes moyennes dans la partie orientale.Cette configuration s'explique par la partition de la Pologne entre les Empires austrohongrois, allemand et russe de la fin du XVIIIème au début du XXème siècle, qui menaient des politiques urbaines très différentes (Trzeciakowski, 1981). De plus, le contrôle des migrations entre les villes de la période socialiste et la répartition de la population au plus près des ressources minières dans des villes nouvelles a eu un 
impact sur cette configuration (Coudroy de Lille, 2007 ; Zborowski, 2009). Cependant un taux de croissance relativement plus élevé des villes à l'Est depuis 1991, contribue à l'argument d'un certain rééquilibrage. En effet, les villes moyennes de l'Est du pays ont su sortir de la crise des années 1990 en se tournant vers le secteur des services ou de l'innovation (WĘcławowicz, 2010) et en se caractérisant par un potentiel de développement métropolitain futur. Rzeszów, ayant connu la revitalisation de son ancien pôle industriel et d'innovation du secteur aérien et militaire, en est un exemple (Noworól et al., 2010). Les grandes villes dont l'industrialisation remonte au XIXème siècle, comme Łódź, ont connu un déclin dès les années 1980, annonçant la décadence du régime, puis son effondrement en 1989 (Gwosdz, 2004 ; WĘcławowicz, 2010).

Ainsi, l'étude de la hiérarchie témoigne d'un système de villes en Pologne de plus en plus polycentrique, ce qui s'explique par la persistance du poids important et équivalent au cours du temps des deux premières villes (Gwosdz, 2004). Cependant le rééquilibrage général depuis 1991 est à interpréter avec précaution, car il correspond au début de la périurbanisation des années 1990 en Pologne (Śleszyński, 2006). Ainsi ce résultat pourrait venir s'expliquer aussi par la forte croissance des petites villes dans les périphéries des grandes agglomérations. De plus la trame urbaine du pays parait plutôt inégalitaire avec une concentration des grands pôles à l'ouest du territoire. Ainsi si l'on prend en compte les deux critères morphologiques définis par le projet ESPON 1.1.1, une polycentricité morphologique élevée serait à rediscuter, ce qui rejoint d'autres travaux pour les années 2000 (Komornicki et. al., 2013 ; Pyka, 2011).

Nous allons donc étudier dans la partie suivante quelles villes polonaises sont les plus impliquées dans les réseaux métropolitains et économiques mondiaux.

\section{Connectivités économiques des villes polonaises avec le reste du monde depuis 2004}

\section{A. Les villes polonaises dans les réseaux d'entreprises mondiaux en} 2004 et 2013

Les connectivités entrepreneuriales entre villes polonaises et étrangères permettent de comprendre comment s'opère la métropolisation dans l'ensemble du système de villes polonais. Deux bases de données ont été mobilisées : Połączenia Świat (Connections Monde) ${ }^{24}$ sur 1242 entreprises en Pologne en 2004 et Orbis (Bureau Van Dick) ${ }^{25}$ sur 1290 entreprises en 2013. Celles-ci renseignent sur leur localisation dans les villes, mais également sur celles des firmes étrangères qui détiennent leur capital (Albert-Bohan, 2015 ; Rozenblat, Di Lello, 2014 ; Śleszyński, 2007) ${ }^{26}$. Après agrégation, nous pourrons dégager les villes accueillant le plus d'entreprises et jouant le rôle d'interfaces avec les autres métropoles mondiales. 
Figure 5 : Nombre d'entreprises polonaises contrôlées à plus de $50 \%$ par les institutions étrangères mondiales en 2004 et 2013 et situées dans les agglomérations

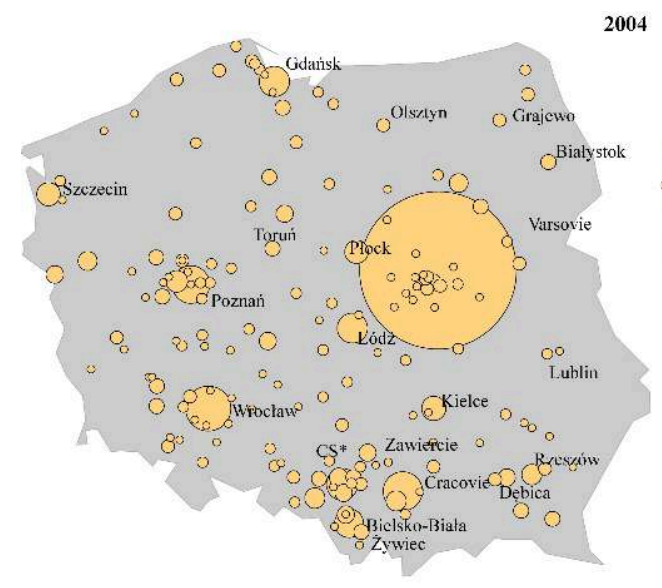

Source : Stescyinski, 2007 es base de données Orbis, 201

2004

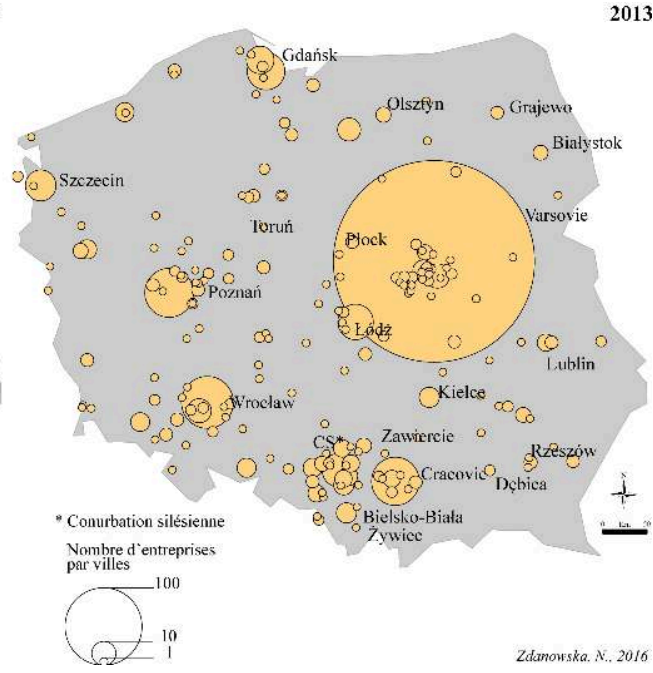

La figure 5 met en avant une extrême polarisation à Varsovie de la localisation des entreprises détenues par les firmes étrangères en 2004 et $2013^{27}$. En 2013, la capitale concentre $45 \%$ du capital étranger investi dans le pays. Cette concentration est l'héritage du système socialiste, où toutes les fonctions politiques et administratives les plus importantes étaient localisées à Varsovie (Śleszyński, 2015). Le reste des entreprises contrôlées par l'étranger sont situées plutôt à l'Ouest, dans la conurbation silésienne, à Cracovie, Wrocław, Poznań et Gdańsk et dans une moindre mesure sur la façade Est à Grajewo, Białystok, Olsztyn, Lublin ou Rzeszów.

En 2013, le capital des entreprises en Pologne est détenu à plus de 65\% par les firmes de l'Europe de l'Ouest ${ }^{28}$ et majoritairement par les institutions allemandes du secteur automobile (Volkswagen AG), bancaire (Deutsche Bank AG, Commerzbank AG), de sidérurgie (ThyssenKruppAG) et de construction (filiale allemande de Strabag AG). 
Figure 6 : Liens de contrôle de plus de $50 \%$ du capital des entreprises polonaises par les entreprises allemandes situées dans les agglomérations en 2013

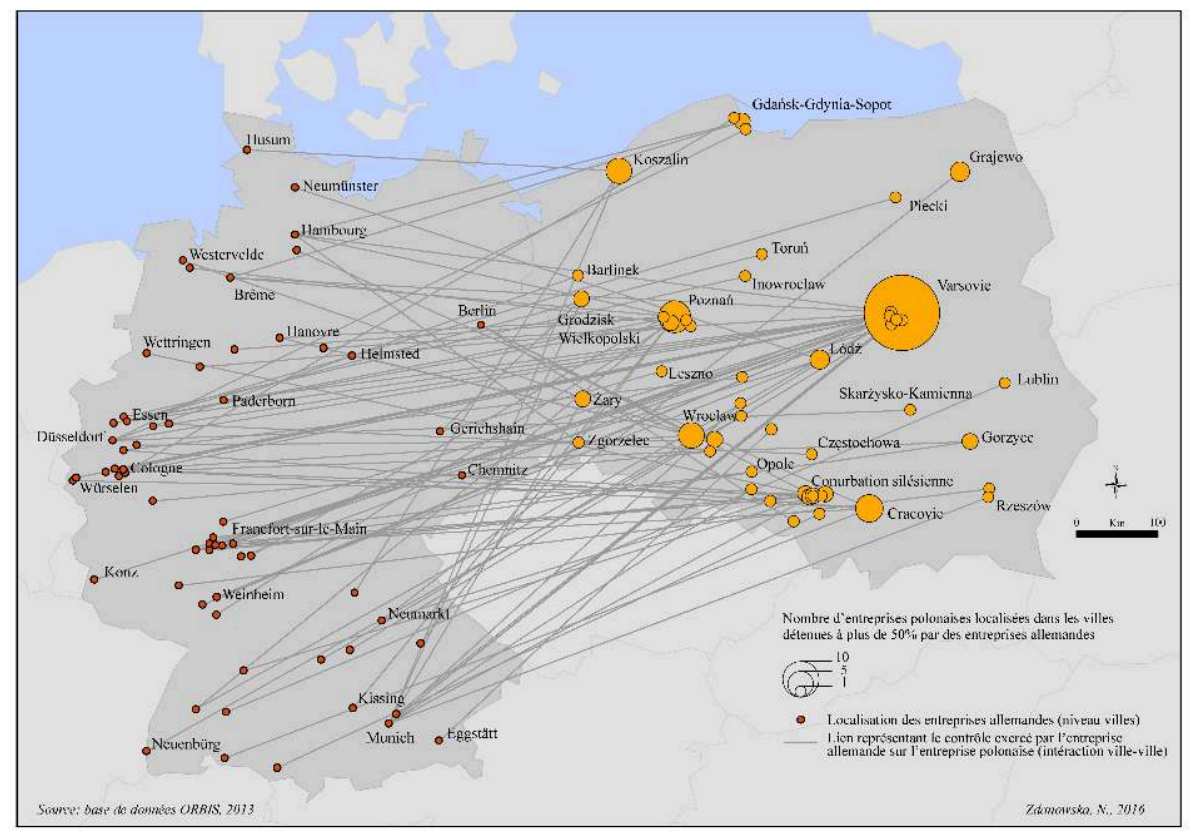

Les entreprises détenues par les firmes allemandes se concentrent à Varsovie, Poznań, Cracovie, Wrocław, Gdańsk et dans d'autres villes de taille moins importantes comme Grodzisk Wielkopolski, Barlinek, Koszalin, majoritairement dans la partie Nord-Ouest du pays. En effet elles correspondent aux anciennes zones d'influences germaniques qui témoignent d'une tradition d'échanges qui perdure jusqu'à aujourd'hui (Lepesant, 2011). Cette dissymétrie Est-Ouest, certes justifiée par des liens historiques et la proximité géographique, reste une observation importante car l'Allemagne est le pays exerçant le plus de contrôle sur les entreprises en Pologne. Le contrôle provenant de l'Ukraine, la Biélorussie et la Russie représente en 2013 seulement $2 \%$ et se concentre surtout à Płock et Varsovie ${ }^{29}$.

L'analyse des figures 5 et 6 révèle un déséquilibre au sein du système de villes polonais quant à l'insertion des villes dans les réseaux d'entreprises mondiaux. La polarisation des fonctions économiques par Varsovie (Śleszyński, 2007, 2013) permet de déduire que seule la capitale et quelques autres villes de taille moyenne disposent aujourd'hui de fonctions métropolitaines et d'une capacité d'ouverture vers l'étranger. En effet le phénomène de métropolisation a concerné en premier lieu Varsovie dès les années 1990 (Bourdeau-Lepage, 2004, 2004a). Les autres villes peinent encore jusqu'à aujourd'hui à faire partie de ce processus (Bourdeau-Lepage, 2007). Malgré leur dynamique démographique depuis 2001, relevée dans la partie précédente, les villes de l'Est sont moins connectées avec d'autres métropoles. Ainsi il semble difficile de parler d'une polycentricité en termes de connectivités entrepreneuriales. 


\section{B. Les villes polonaises dans les réseaux commerciaux en 2005 et} 2011

La participation des villes polonaises dans les réseaux d'échanges commerciaux reflète l'acquisition de fonctions économiques et de décisions d'ordre international spécifique à la métropolisation (Komornicki et. al., 2013 ; Komornicki, Szejgiec, 2015 ; Vandermotten et al., 2010).

La base de données Handel Zagraniczny (Commerce International) du Ministère des Finances de Pologne sur les échanges commerciaux entre villes polonaises et les pays de monde en 2005 et 2011 (Komornicki, Szejgiec, 2015) a permis de renseigner de façon exceptionnelle les exportations partant des villes polonaise ${ }^{30}$ vers les pays destinataires du monde entier. Eneffet dans la grande majorité des cas, ces données ne sont accessibles qu'à l'échelle nationale.

Figure 7 : Taux de croissance annuel moyen (2005-2011) des exportations polonaises exprimées en Euros partant des villes à statut de powiat vers les pays du monde entier

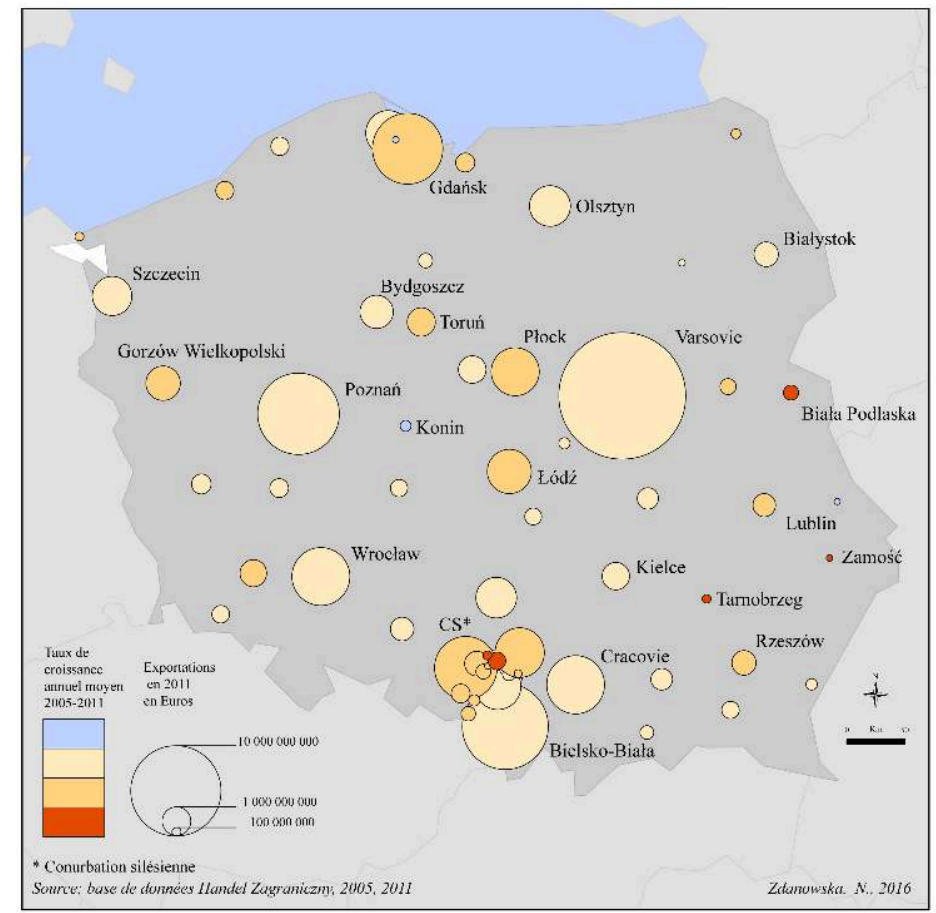

La figure 8 révèle que les exportations partent majoritairement de Varsovie, Cracovie, de la conurbation silésienne et de Gdańsk (Komornicki et al., 2013). Elles forment selon les spécialistes un "réseau urbain en forme d'hexagone" au Centre-Ouest du pays (Komornicki, Szejgiec, 2015, p. 174). Toute la partie Est est mise à l'écart et représente une valeur moindre en termes de valeurs absolues des exportations (Chiappini, 2010). Cependant certaines petites villes comme Biała Podlaska, Zamość ou Tarnobrzeg, voire plus grandes comme Lublin et Rzeszów, présentent entre 2005 et 2011 un taux de croissance des exportations plus élevé que les villes de «l'hexagone». Ces exportations s'orientent vers l'Ukraine et la Biélorussie, ce qui reflète une réactivation des traditions 
d'échanges datant du socialisme ou même de périodes antérieures (Lepesant, 2011 ; Komornicki, 2004).

Ainsi on observe un déséquilibre entre villes polonaises de «l'hexagone » et le reste des villes quant à leur insertion en tant que métropoles dans les réseaux commerciaux mondiaux. Cela permet de remettre à nouveau en question la polycentricité élevée du système urbain polonais annoncée par le projet ESPON 1.1.1. Malgré cette dissymétrie, l'observation des exportations partant des villes de l'Est de la Pologne reste intéressante. Elle pourrait témoigner d'un potentiel de dynamisme futur de ces villes qui auraient leur rôle à jouer dans un réseau tourné vers la façade Est de l'Union européenne.

\section{Conclusion}

Le présent article a visé à analyser la dynamique démographique des villes polonaises et à évaluer leur insertion dans les réseaux économiques mondiaux, afin de comprendre comment s'opère le processus de métropolisation dans le système urbain en Pologne. Est-il possible de parler d'un système de ville polycentrique en Pologne aujourd'hui?

L'évolution de la distribution des tailles des villes montre que les inégalités entre villes tendent à se réduire entre 1991 et 2011, permettant de mettre en évidence un rééquilibrage en termes de hiérarchie urbaine. Cependant, l'analyse de la trame urbaine révèle une dissymétrie profonde dans le système de villes entre l'Est et l'Ouest du pays. Ainsi le système urbain polonais est de plus en plus polycentrique du point de vue de la distribution des tailles de villes. Cependant cette observation peut être relativisée lorsque l'on considère la répartition des villes dans le territoire.

De plus l'insertion inégale des villes polonaises dans les réseaux mondiaux d'entreprises et commerciaux observée à partir du milieu des années 2000, permet d'envisager une forme monocentrique plutôt que polycentrique du système de villes polonais en termes de relations économiques avec l'étranger. En effet, peu de villes à part la capitale accueillent des entreprises ayant des relations majeures avec l'étranger. De plus ces entreprises sont implantées de manière inégale sur l'ensemble du territoire, majoritairement à l'ouest du pays. En outre, les exportations partent majoritairement des grandes villes et excluent de ces réseaux commerciaux de nombreuses villes de l'Est du territoire.

Ces résultats permettent de constater que l'accès aux fonctions métropolitaines des villes en Pologne s'opère de manière très inégale et sélective depuis 1990 (BourdeauLepage, 2004a), ce qui rejoint par définition la caractéristique majeure de la métropolisation (Bretagnolle, Le Goix, Vacchiani-Marcuzzo, 2011). Cependant comme ce phénomène a débuté plus tard qu'en Europe de l'Ouest, les villes centre-européennes ont dû associer le rattrapage économique et l'insertion dans un réseau de métropoles déjà très concurrentiel (Zdanowska, 2015). Pour cette raison ce sont d'abord les capitales qui ont connu le phénomène de métropolisation dans les années 1990 (Bourdeau-Lepage, 2004a). Ce déséquilibre métropolitain persiste jusqu'à aujourd'hui et a même tendance à s'accroitre ce qui laisse à réfléchir quant au sort des villes petites et moyennes, spécifiquement dans la façade Est du pays. 


\section{BIBLIOGRAPHY}

Albert-Bohan C., 2015, Les stratégies des firmes multinationales automobiles dans les villes de l'élargissement européen : réseaux urbains et organisation en chaine globale de valeur, Thèse de doctorat, Université de Lausanne, 355 p.

Bourdeau-Lepage L., 2004 " Varsovie une nouvelle métropole », GaWC Research Bulletin (en ligne), $\mathrm{n}^{\circ}$ 142, mis en ligne le 23 avril 2004, consulté le 2 novembre 2014, URL : http:// www.lboro.ac.uk/gawc/rb/rb142.html

Bourdeau-Lepage L., 2004a, « Metropolization in Central and Eastern Europe: Unequal Chances », GaWC Research Bulletin, $\mathrm{n}^{\circ}$ 141, mis en ligne le 23 avril 2004, consulté le 19 avril 2016, URL: http:// www.lboro.ac.uk/gawc/rb/rb141.html

Bourdeau-Lepage L., 2007 « Advanced services and city globalization on the Eastern fringe of Europe », Belgeo n 1, mis en ligne le 09 décembre 2013, consulté le 19 April 2016, URL : http:// belgeo.revues.org/11703.

Bourdeau-Lepage L., Huriot J-M., 2005 « The metropolis in retrospect, From trading metropolis to the global metropolis » Recherches économiques de Louvain, vol. 71, n³, p. 257-282.

Bretagnolle A., 2009, Villes et réseaux de transport : des interactions dans la longue durée (France, Europe, États-Unis), Habilitation à diriger des recherches, vol. 3, Université Paris 1 PanthéonSorbonne, $204 \mathrm{p}$

Bretagnolle A., 2013, « La ville mondiale : une histoire de représentations », Questions Internationales, Dossier « Les villes mondiales ", $n^{\circ} 60$, Mars-Avril, La Documentation Française, $\mathrm{p}$. $8-20$.

Bretagnolle A., 2015 « La naissance du périurbain comme catégorie statistique en France. Une perspective internationale ", L'Espace Géographique, n¹, p. 18-37.

Bretagnolle A., Le Goix R., Vacchiani-Marcuzzo C., 2011, « Métropoles et mondialisation », Documentation photographique, La Documentation française, $\mathrm{n}^{\circ} 8082,9 \mathrm{p}$.

Bretagnolle A., Giraud T., Guérois M., Mathian H., 2012, « A new database for the cities of Europe? Urban Morphological Zones (CLC2000) confronted to three national databases of urban agglomerations (Denmark, France, Sweden) », Environment and Planning B, vol. 39, n³, p. 439-458.

Bretagnolle A., Guérois M., Pavard A., Gourdon P., Zdanowska N., Lizzi L., 2016, Demographical Trajectories of European urban areas (1961-2011) (TRADEVE), rapport de recherche, URL : https:// halshs.archives-ouvertes.fr/halshs-01311420.

Bretagnolle A., Pumain D., Vacchiani-Marcuzzo C., 2007, « Les formes des systèmes de villes dans le monde » in Mattei M.-F., Pumain D., Données urbaines, 5, Paris, Anthropos-Economica, p. 301314.

Brunet R., Ferras R., Théry H., 1993, Les mots de la géographie, dictionnaire critique, Paris, Reclus, La Documentation française, $518 \mathrm{p}$.

Cattan N., Saint-Julien T., 1998 « Modèles d'intégration spatiale et réseau des villes en Europe occidentale », Espace géographique, vol. 27, p. 1-10.

Chiappini R., 2010, Dynamique des spécialisations et performances commerciales des pays d'Europe Centrale et Orientale (PECO), LAREFI Working Paper CR10-EFI/05, 41 p., URL : http://lare-efi.ubordeaux4.fr/IMG/pdf/CR10-EFI_05.pdf 
Coudroy de Lille L., 2007, « Nowe Tychy, ville satellite de la conurbation silésienne », Regards sur l'Est, Dossiers : "Les Villes nouvelles à l'Est", mis en ligne le 15 novembre 2007, consulté le 27 avril 2016, URL : http://www.regard-est.com/home/breve_contenu.php?id=792

Dijkstra L., Poelman H., 2012, « Cities in Europe. The new OECD-EC definition. Regional Focus », RF 01/2012, Regional and Urban Policy, 15 p.

De Backer K., Menghinello S., Pinto Ribeiro S., 2010, The OECD ORBIS database: responding to the need for firm-level micro-data in the OECD, OECD Working paper, URL: http://www.oecd.org/ officialdocuments/publicdisplaydocumentpdf/?doclanguage $=$ en \& cote $=$ std $/$ doc $\% 282010 \% 291$

EEA, 2002, CORINE land cover update, Technical guidelines, EEA, 56 p., URL: http:// www.eea.europa.eu/publications/technical_report_2002_89

ESPON project 1.1.1, 2005, Potentials for polycentric development in Europe, report, ESPON Monitoring Committee, 1000 p., URL: http://www.espon.eu/export/sites/default/Documents/ Projects/ESPON2006Projects/ThematicProjects/Polycentricity/fr-1.1.1_revised-full.pdf

ESPON project 1.4.3, 2007, Study on Urban Functions, final report, ESPON Monitoring Committee, 253 p., URL :https://www.espon.eu/export/sites/default/Documents/Projects/ ESPON2006Projects/StudiesScientificSupportProjects/UrbanFunctions/fr-1.4.3_April2007final.pdf

Gawryszewski A., 1974, ZwiĄzki przestrzenne miĘdzy migracjami stałymi i dojazdami do pracy oraz czynniki przemieszczeń ludności (Relation spatiales entre migrations et navettes domicile-travail ainsi que les détérminants des déplacements de la population), Wrocław, Prace Geograficzne, IG PAN, nr 109, 155 p.

Geppert A., 2009, « Polycentricity: can we make it happen? From a concept to its implementation ", Urban Research and Practice, vol. 3, n³.

Gorzelak G., Jałowiecki B., SmĘtkowski M., 2009, « Obszary metropolitarne w Polsce: problemy rozwojowe i delimitacja », EUROREG, Raporty i analizy, 1/2009.

Gwosdz K., 2004, Ewolucja rangi miejscowości w konurbacji przemysłowej. Przypadek Górnego ŚlĄska (Evolution du rang des localités dans une conurbation industrielle. Cas de la Haute Silésie), Kraków, Instytut Geografii i Gospodarki Przestrzennej Uniwersytetu Jagiellońskiego.

Jałowiecki B., 2005, « Polskie miasta w procesie metropolizacji », Studia Regionalne i Lokalne, nº 1(19), p. 1-14.

Komornicki T., 2004, « Obszary koncentracji eksportu w przestrzeni przemysłowej Polski » (Zones de concentration des exportations dans l'espace industriel de la Pologne), Przegląd Geograficzny, vol. 76, nº 4, p. 473-491.

Komornicki T., Korcelli P., Siłka P., Śleszyński P., ŚwiĄtek D., 2013, PowiĄzania funkcjonalne pomiĘdzy polskimi metropoliami (Relations fonctionnelles entre métropoles polonaises), Warszawa, SEDNO, $215 \mathrm{p}$.

Komornicki T., Szejgiec B., 2015, « Spatial differentiation of Polish export linkages », Geographia Polonica, vol. 88, ํㅡ 1, p. 173-179.

Korcelli-Olejniczak E., 2004, Funkcje metropolitarne Berlina i Warszawy w latach 1990-2002. Wspótzależności pozycji w systemie miast Europy Środkowej (Fonctions métropolitaines de Berlin et Varsovie dans les années 1990-2002. Interdépendances des positions dans le système de villes d'Europe centrale), Prace Geograficzne nํㅜ 198, Warszawa, Polska Akademia Nauk, Instytut Geografii i Przestrzennego Zagospodarowania. 
Kotela C., 1990, « Gospodarka przestrzenna w GOP na przykładzie realizacji planów zagospodarowania przestrzennego w latach 1945-1989» (Economie géographique de la région industrielle de Haute Silésie sur l'exemple de la réalisation des plans d'aménagement du territoire dans les années 1945-1989), Śląski Kwartalnik Urbanistyki i Architektury,nr.1-2, p. 11-27.

Le Gléau J.-P., Pumain D., Saint-Julien T., 1996, « Villes d'Europe : à chacun sa définition », Economie et Statistique, oㅡ 294-295, 4/5, p. 9-23.

Lepesant G., 2011, Géographie économique de l'Europe centrale. Les nouveau territoires européens, Paris, Presses de Sciences Po, $352 \mathrm{p}$.

Lijewski T., 1958, « Rozwój sieci kolejowej woj. Warszawskiego » (Développement du réseau de chemin de fer de la voïvodie de Varsovie), Przegląd geograficzny, nr. 3, p. 461-477.

Michalet C.-A., 2007, Mondialisation, la grande rupture,Paris,La Découverte, 167 p.

Moriconi-Ebrard F., 1994, Géopolis. Pour comparer les villes du monde, Paris, Anthropos, Economica, Collection Villes, 246 p.

Noworól A., Noworól K., Hałat P., 2010, Program Rewitalizacji Obszarów Miejskich w Rzeszowie na lata 2007-2015 (Programme de revitalisation des aires urbaines à Rzeszów pour les années 2007-2015), rapport, Alexander Noworól Konsulting.

ORATE 1.1.1, 2005, « Les potentiels de développement polycentrique en Europe », Rapport final du projet, 28 p., URL : http://dgo4.spw.wallonie.be/DGATLP/DGATLP/Pages/DAU/Dwnld/ORATEESPON/ORATE\%20ESPON\%20111\%20F.pdf

Parr J. B., 2007, « Spatial definitions of the city: four perspectives », Urban Studies, 44, p. 381-392.

Peeters D., 2011, The functional Urban Areas database, Technical Report, Rapport final, phase 1, ESPON Data Base 2013, 193 p.

Pumain D., 2007, « Lois d'échelle et mesure des inégalités en géographie », Revue européenne des sciences sociales, tome XLV, $\mathrm{n}^{\circ}$ 3, p. 55-65.

Pumain D., Saint-Julien T., 1996 (dir.), Urban networks in Europe, Paris, John Libbey-INED, Congresses and Colloquia, 15, $252 \mathrm{p}$.

Rozenblat C., Di Lello O., 2014« Les réseaux de firmes multinationales dans les villes d'Europe centre-orientale », Cybergeo : European Journal of Geography, Espace, Société, Territoire, document 678, mis en ligne le 20 juin 2014, consulté le 21 avril 2016, URL : http:// cybergeo.revues.org/26325

Rozenblat C., Pumain D., 2007, "Firm linkages, innovation and the evolution of urban systems", in Taylor, P. J. et al., Cities in Globalization. Practices, policies and theories, London, Routledge, p. 130-156.

Pyka R., 2011, « La métropolisation en Pologne : le fonctionnement et l'avenir des espaces métropolitains polonais ",Métropoles(en ligne), vol. 10, mis en ligne le 31 décembre 2011, consulté le 04 décembre 2014, URL : http://metropoles.revues.org/4515

Saint-Julien T., 1981, «Croissance industrielle et trame urbaine », Bulletin de l'Association de géographes français, vol. 58, n475-476, p. 59-66.

Sassen S., 1991, The Global City, Princeton, NJ, Princeton University Press, 447 p.

Śleszyński P., 2006, « Suburbanization processes in Poland after 1989 »ProblemsofGeography, n²1-2, p. 105-117. 
Śleszyński P., 2007, Gospodarcze funkcje kontrolne w przestrzeni Polski (Fonctions économiques de contrôle en Pologne), Warszawa, Instytut Geografii i Przestrzennego Zagospodarowania, Polska Akademia Nauk, 284 p.

Śleszyński P., 2013, « Demographic change in the functional urban areas in Poland, 2000-2010 ", Geographia Polonica, vol. 86, n² 2, p. 169-171.

Śleszyński P., 2015, « Economic control functions in Poland in 2013 », Geographia Polonica, vol. 88, $\mathrm{n}^{\circ} 4$, p. 701-708.

Taylor P. J., 2003, « European Cities in the World City Networks », GaWC Research Bulletin, n 105.

Taylor P. J., 2004, World city network: a global urban analysis, London, Routledge, 256 p.

Trzeciakowski L., 1981, Ziemie polskie pod panowaniem państw zaborczych (1815-1918), \{Terres polonaises sous la répartition (1815-1918)\}, in Topolski J., Dzieje Polski (Histoire de la Pologne), Warszawa, PWN, p. 445-610.

Vandermotten C., Vermoesen F., De Lannoy W., De Corte S., 1999, Villes d'Europe. Cartographie comparative, Bruxelles, Bulletin du Crédit Communal, nº 207-208, 408 p.

Vandermotten C., Marissal P., Van Hamme G., 2010, La production des espaces économiques. La formation du système-monde, tome 1, Bruxelles, Université Libre de Bruxelles, $426 \mathrm{p}$.

WĘcławowicz G., 2010, Charakterystyka głównych trendów i zmian w rozwoju obszarów miejskich Polski (Caractéristiques des principales tendances et changements de développement des zones urbaines en Pologne), rapport, Ministerstwo Rozwoju Regionalnego, Departament Koordynacji Polityki Strukturalnej, 201 p.

Zborowski A., 2009, « Zmiany zasiĘgu oddziaływania miast w Polsce w okresie transformacji systemowej, na przykładzie dojazdów do pracy » (Changements des zones d'influence des villes en Pologne pendant la transformation du système, sur l'exemple des navetteurs), in Górka Z., Zborowski A., Człowiek i rolnictwo, Instytut Geografii i Gospodarki Przestrzennej, Kraków, Uniwersytet Jagielloński, p. 249-263.

Zdanowska N., 2015, « Metropolisation and the evolution of systems of cities in the Czech Republic, Hungary and Poland since $1950 »$, Deturope - The Central European Journal of Regional Development and Tourism, vol. 7, n 2, p. 45-64, URL: http://www.deturope.eu/file_download.php? type $=2 \&$ item $=116$

\section{NOTES}

1. "A metropolis is a city, but it is more than a city ».

2. Pays postsocialistes qui sont rentrés dans l'Union européenne depuis 2004.

3. Globalization and World Cities, Université de Loughborough.

4. Varsovie, conurbation Silésienne, Cracovie, Poznań, Gdańsk-Gdynia-Sopot, Wrocław, Łódź (Pyka, 2011).

5. Pour la France douze secteurs caractériseraient les fonctions métropolitaines (art, banques et assurances, commerce de gros, commercial industriel, gestion, information, informatique, recherche industrielle, recherche publique, services, télécommunications, transports) (Pumain, Saint Julien, 1996).

6. « Koncepcja polityki przestrzennego zagospodarowana kraju» (Conception de la politique spatiale d'aménagement du territoire), 2001.

7. Narodowy Plan Rozwoju 2007-2013 (NPR). 
8. Dans cet article cette conurbation est considérée comme composée des villes de Katowice, Sosnowiec, Gliwice, Bytom, Zabrze et Ruda Śląska. L'auteur est conscient de la multitude de définitions possibles.

9. MEGA: Metropolitan Economic Growth Areas (ESPON project 1. 1. 1, Potentials for polycentric development in Europe, 2005) (Potentiels de développement polycentrique en Europe).

10. ESPON project 1.1.1, Potentials for polycentric development in Europe, 2005.

11. ORATE 1.1.1, Les potentiels de développement polycentrique en Europe, 2005.

12. L'analyse s'inscrit dans le cadre d'une thèse portant sur l'intégration spatiale des systèmes de villes en Europe Centrale et Orientale par les réseaux d'entreprises, commerciaux et aériens depuis 1989.

13. Nous reprenons ici les deux premiers critères morphologiques de polycentricité élaborés pas les auteurs du projet ESPON 1. 1. 1., sans prendre en compte le troisième sur les connectivités liées au transport entre villes polonaises.

14. Le projet TRADEVE «TRAjectoires DEmographiques des Villes Européennes » a été financé par l'Université Paris 1 Panthéon-Sorbonne pour la période 2014-2016. L'objectif a été de construire une base de données harmonisée sur les délimitations et populations des agglomérations urbaines en Europe de 1961 à 2011. La disponibilité des données a permis de remonter jusqu'à 1961.

15. La base a été acquise pour les pays d'Europe centrale et orientale en 2010 et 2013 grâce à C. Rozenblat dans le cadre d'une convention de recherche.

16. L'auteur a effectué des missions de terrains en 2015 en Bulgarie, Croatie, Hongrie, République tchèque, Roumanie, Slovaquie et Slovénie, où ces données ne sont accessibles qu'à l'échelle nationale.

17. Projet de loi sur l'administration municipale du 8 mars 1990, Conseil des Ministres de l'Assemblée Nationale de la République de Pologne.

18. Construits en combinant un critère de densité minimale de 650 habitants par $\mathrm{km}^{2}$ des Morphological Urban Areas et un critère fonctionnel de navettes domiciles-travail (Peeters, 2011).

19. Selon Eurostat, une LAU2 (Local Administrative Unit) correspond à la plus petite division administrative en Europe.

20. Pour la justification des critères, voir le rapport du projet. Elle résulte notamment d'une confirmation auprès de géographes à Budapest, Prague, Bucarest, Bratislava, Zagreb, Ljubljana et Sofia rencontrés par l'auteur en 2015.

21. La taille conséquente de certaines agglomérations comme Poznań est due à la surface importante des LAU2 dans cette partie du pays.

22. Polska Rzeczypospolita Ludowa.

23. Le choix des classes repose sur une comparaison absolue en mettant en œuvre une discrétisation de type progression géométrique sur les distributions réunies.

24. Construite par P. Śleszyński à partir de la base Hoppenstedt Bonnier (Śleszyński, 2007).

25. Explorée par l'équipe de C. Rozenblat de l'Université de Lausanne et par l'OCDE (De Backer et al., 2010).

26. Les deux bases indiquent les noms, les coordonnées géographiques et les chiffres d'affaires respectivement des entreprises possédées et possédantes (Śleszyński, 2007 ; Albert-Bohan, 2015).

27. L'échantillon des entreprises est plus ou moins équivalent. Cependant il n'est pas possible de comparer les deux années et d'émettre des conclusions quant à la hiérarchisation des fonctions métropolitaines dans quelques villes. Cependant des travaux récents confirment leur concentration à Varsovie sur la même période (Śleszyński, 2015).

28. Pays européens hors ceux ayant adhéré à l'Union européenne depuis 2004.

29. La base de données ORBIS reste imparfaite, même si 50\% des lacunes dans les adresses des entreprises ont pu être corrigées par l'auteur. 
30. Il s'agit de villes à statut de « powiat » correspondants aux capitales des powiat (district) division territoriale intermédiaire entre la voïvodie (région administrative) et la gmina (commune).

\section{ABSTRACTS}

This article examines the relationship between metropolisation and the evolution of the system of cities in Poland. Central and Eastern European cities have only started experiencing the process of metropolisation since the fall of the Berlin Wall in 1989. Yet this period corresponds to an intense globalisation of the economy which has been characterised by some as an emergence of a world urban system. While the metropolises of formerly industrialised countries had already been strongly interconnected on a macro-regional level, Central and Eastern European countries took on the unique approach of prioritising relations directly at an international level. In addition to its highly exacerbated characteristic, metropolisation in Central and Eastern Europe has been taking place within a substantially shorter time period relative to other countries. As far as the impact of this process on the dynamics of development of Central and Eastern European system of cities is concerned, Poland represents a particular case due to its relative polycentricity since 1960 .

In this article, we will examine, the evolution of the Polish system of cities by using demographic and functional criteria based on several national databases. Results show that the economic connectivities between cities, which have become the key features of the metropolitan phenomena nowadays, put into perspective the idea of an egalitarian or polycentric urban system in Poland.

L'article vise à étudier la relation entre la métropolisation et l'évolution du système de villes en Pologne. Les villes des pays d'Europe Centrale et Orientale n'ont connu le processus de métropolisation qu'à partir de la chute du mur de Berlin en 1989. Or, il s'agit d'une période de mondialisation intense de l'économie qui se caractérise, selon certains chercheurs, par l'émergence d'un système urbain mondial. Alors que les métropoles des pays anciennement industrialisés étaient déjà fortement interconnectées entre elles à l'échelle macro-régionale, celles des pays d'Europe centre-orientale ont connu une dynamique originale, qui s'est directement inscrite à l'échelon $d u$ monde. En plus de son caractère exacerbé, cette métropolisation a lieu aujourd'hui sur une période nettement plus courte que dans d'autres pays. L'étude de l'influence de ce processus sur la dynamique des systèmes de villes en Europe Centrale et Orientale présente un intérêt tout particulier pour la Pologne, qui se démarque par un caractère relativement polycentrique depuis 1960.

Nous étudions ici l'évolution du système de villes à partir de critères démographiques et fonctionnels à l'aide de plusieurs bases de données nationales. Les résultats montrent que les connectivités économiques, les plus caractéristiques du phénomène métropolitain de nos jours, permettent de relativiser l'idée d'un système égalitaire et polycentrique des villes polonaises.

In dem vorliegenden Artikel wird das Verhältnis zwischen der Metropolenbildung und der Entwicklung der Stadtsysteme in Polen untersucht. In zentral- und osteuropäischen Städten ist die Metropolenbildung erst nach dem Fall der Berliner Mauer 1989 eingetreten. Einige Forscher charakterisieren diese Zeit, die durch eine starke Globalisierung der Wirtschaft geprägt ist, mit 
einer Ausdehnung eines weltweiten urbanen Systems. Während Metropolen der früh industrialisierten Länder bereits auf makro-regionaler Ebene stark miteinander verbunden waren, haben zentral- und osteuropäische Städte direkte Beziehungen auf internationaler Ebene aufgebaut. Neben diesen Charakteristika hat die Metropolenbildung in zentral- und osteuropäischen Ländern in wesentlich kürzerer Zeit als in anderen Ländern stattgefunden. Was diesen dynamischen Prozess der Stadtentwicklung in zentral- und osteuropäischen Städten betrifft, stellt Polen eine Besonderheit dar. Seit 1960 ist für Polen eine polyzentrische Entwicklung festzustellen.

Diese Studie untersucht die Entwicklung von polnischen Stadtsystemen hinsichtlich demografischer und funktionaler Kriterien basierend auf mehreren nationalen Datensätzen. Die Ergebnisse zeigen, dass die wirtschaftlichen Zusammenhänge, die heutzutage die Hauptcharakteristika der Metropolenbildung darstellen, die Idee eines egalitären Systems und eines Polyzentrismus der polnischen Städte relativieren.

\section{INDEX}

Mots-clés: métropolisation, monocentricité, Pologne, polycentricité, système de villes Schlüsselwörter: Metropolenbildung, Monozentrismus, Polen, Polyzentrismus, System der Städte/Stadtsysteme

Keywords: metropolisation, monocentricity, Poland, polycentricity, system of cities

\section{AUTHOR}

\section{NATALIA ZDANOWSKA}

Université Paris 1 Panthéon-Sorbonne, UMR 8504 Géographie-cités, 13 rue du Four 75006

Paris, natalia.zdanowska@univ-paris1.fr 